\title{
Efficient coding schemes for low-rate wireless personal area networks
}

ISSN 1751-8628

Received on 13th May 2015

Revised on 7th December 2015

Accepted on 15th February 2016 doi: 10.1049/iet-com.2015.0699 www.ietdl.org

\author{
Hua Qian ${ }^{1,2,3,4 凶}$, Shengchen Dai, 1,2,4,Kai Kang ${ }^{3}$, Xudong Wang ${ }^{5}$ \\ ${ }^{1}$ Shanghai Institute of Microsystem and Information Technology (SIMIT), Chinese Academy of Sciences (CAS), Shanghai, \\ People's Republic of China \\ ${ }^{2}$ Key Laboratory of Wireless Sensor Network and Communication, CAS, Shanghai, People's Republic of China \\ ${ }^{3}$ Shanghai Advanced Research Institute (SARI), CAS, Shanghai, People's Republic of China \\ ${ }^{4}$ Shanghai Research Center for Wireless Communications, Shanghai, People's Republic of China \\ ${ }^{5}$ University of Michigan-Shanghai Jiao Tong University Joint Institute, Shanghai Jiao Tong University, Shanghai, \\ People's Republic of China \\ 凶-mail: qianh@sari.ac.cn
}

\begin{abstract}
The emerging market of Internet of things has created great demand for low-cost, low-power wireless technologies. Existing IEEE 802.15.4 standard is designed for low-rate wireless personal area networks (LR-WPANs). However, current standard does not fully utilise the benefit of the code redundancy. In this study, the authors propose new coding schemes for LR-WPANs with improved coding gain. They first propose a block code based on extended Bose-Chaudhuri-Hocquenghem $(\mathrm{BCH})$ code that increases the minimum Hamming distance compared with the existing code used in LR-WPANs. The computational complexity of the encoder and decoder remains about the same. In addition, by applying the extended $\mathrm{BCH}$ code directly to LR-WPANs, the data rate of the system can be increased without sacrificing coding performance. They further propose a tail-biting convolutional (TBC) code with optimum generator polynomials for LR-WPANs. The proposed TBC code enjoys significant performance improvement while preserving the effective code rate as well as a low decoding complexity. Simulation results validate the effectiveness of the proposed coding schemes.
\end{abstract}

\section{Introduction}

As the Internet of things (IoT) attracts more and more attention recently, low-cost and low-power wireless communications become essential to provide ubiquitous connectivity [1]. Constant-envelope modulation technique and low-rate (LR) coding schemes are adopted to improve power efficiency and system performance of LR wireless personal area networks (LR-WPANs) at different frequency bands. Among all wireless standards, IEEE 802.15.4 standard plays an important role in IoT for LR-WPANs. The $2.4 \mathrm{GHz}$ physical layer (PHY) in IEEE 802.15 .4 standard supports an over-the-air data rate of $250 \mathrm{~kb} / \mathrm{s}$. Direct sequence spread spectrum (DSSS) is applied to improve system performance and the chip rate is $2 \mathrm{Mb} / \mathrm{s}$. The signals are then modulated by offset quadrature phase shift keying (OQPSK) with half-sine pulse shaping filter [2]. Since this modulation is identical to minimum the shift keying (MSK) modulation, the transmitted signals have constant envelope. Such a transmitter has advantage in adopting non-linear power amplifiers and achieving high-power efficiency.

Conventional approaches apply differential detection to detect MSK signals. The performance of such detection schemes is not satisfactory [3-7]. The coherent detection achieves good performance. However, it is sensitive to initial phase error or frequency offset. Dai et al. [8] proposed a robust detector, which provides satisfactory performance with only $1 \mathrm{~dB}$ loss compared with the coherent detector in additive white Gaussian noise (AWGN) channel.

In addition to improve the performance of demodulation schemes, the overall system performance can be further improved by fully utilising the benefit of the code redundancy. For example, DSSS in IEEE 802.15.4 standard is not simple spreading. It provides coding gain in addition to spreading gain. The symbol-to-chip mapping can be viewed as a $(32,4)$ block code. The minimum Hamming distance of this code is 12 . The coding gain is $\sim 2 \mathrm{~dB}$ at packet error rate (PER) of $1 \%$ [9].

From channel coding perspective, the coding scheme provided in current standard is not optimal. For general block codes, Griesmer [10] suggested an upper bound of minimum Hamming distance of the codes. The minimum Hamming distance must be $\leq 16$ for a $(32,4)$ code, suggesting that extra coding gain is possible. For convolutional codes, the bound of free distance listed in [11] limits the system performance. The convolutional codes with maximum free distance can be utilised to improve performance. In a word, there is plenty of room to improve the code efficiency for LR-WPANs.

In this paper, we propose new coding schemes with improved coding gain for LR-WPANs. We propose a block code based on extended Bose-Chaudhuri-Hocquenghem $(\mathrm{BCH})$ code that improves the coding gain. Such benefit is obtained without extra computational complexity. By applying the extended $\mathrm{BCH}$ code directly to LR-WPANs, the data rate can be increased without sacrificing the coding performance. To obtain additional coding gain, we further propose a tail-biting convolutional (TBC) code with optimum generator polynomials for LR-WPANs. System performance can be significantly improved.

The rest of this paper is organised as follows. Section 2 provides system setup for LR-WPANs. We take the coding scheme of the 2.4 $\mathrm{GHz} \mathrm{PHY}$ in IEEE 802.15.4 standard as an example for further discussion. Section 3 proposes new coding schemes based on block codes. The proposed coding scheme based on convolutional codes is presented in Section 4. Simulation results are provided in Section 5 that demonstrates the effectiveness of the proposed coding schemes. Section 6 extends the proposed coding schemes to other LR-WPANs standards. Finally, Section 7 concludes this paper. 


\section{System setup}

In this section, we provide a system setup for LR-WPANs. Fig. 1 shows the transceiver structure of general LR-WPANs. An LR encoder is applied to improve the receive sensitivity. The coded bits are then modulated to transmitted signals. To meet the spectral mask, pulse shaping filter is applied. The digital baseband signals are then transformed to analogue domain by digital-to-analogue converter. For a single-carrier, narrow-band system in the applications of LR-WPANs, AWGN channel is reasonable assumption. At the receiver side, the baseband analogue signals are sampled to digital signals by analogue-to-digital converter. Matched filter is utilised to maximise the signal-to-noise ratio (SNR) of the received signals. After synchronisation, the receiver demodulates and decodes the signals.

Channel coding is essential to improve the system performance in LR-WPANs. In this paper, we take the coding scheme of the 2.4 $\mathrm{GHz} \mathrm{PHY}$ in IEEE 802.15.4 standard as an example for further discussion. The encoder for IEEE 802.15.4 standard is shown in Fig. 2. The information bits are segmented into symbols every 4 bits. The symbol $\boldsymbol{u}=\left(u_{0}, u_{1}, u_{2}, u_{3}\right)$ is spread to a 32 bit chip sequence, which is denoted by $c=\left(c_{0}, c_{1}, \ldots, c_{31}\right)$. Table 1 is the symbol-to-chip mapping table in IEEE 802.15.4 standard. OQPSK with half-sine pulse shaping filter is applied. Such modulation is identical to MSK modulation. Since the analogue transmitted signals have constant envelope, power-efficient non-linear power amplifiers can be applied in the transmitter.

The encoder used in the standard is not simple spreading. It provides coding gain. The coding gain for a coded binary OQPSK system can be approximated by [12]

$$
\gamma \simeq 10 \log _{10}\left(R_{\mathrm{c}} d_{\min }-\frac{k \ln 2}{E_{\mathrm{b}} / N_{0}}\right),
$$

where $k$ is the length of information bit sequence, $n$ is the length of codewords, $d_{\min }$ is the minimum Hamming distance of the set of codewords, $R_{\mathrm{c}}=k / n$ is the code rate and $E_{\mathrm{b}} / N_{0}$ is the SNR per bit. In IEEE 802.15.4 standard, the encoder maps every four information bits to a 32 bit chip sequence. The minimum Hamming distance of the set of codewords is 12 . The coding gain is about $2 \mathrm{~dB}$ [9]. On the other hand, this set of codewords is not optimised for coding gain.

\section{Coding schemes based on linear block codes}

Since linear block codes are easy to implement and analyse, and their performances are similar to that of general block codes, we focus on

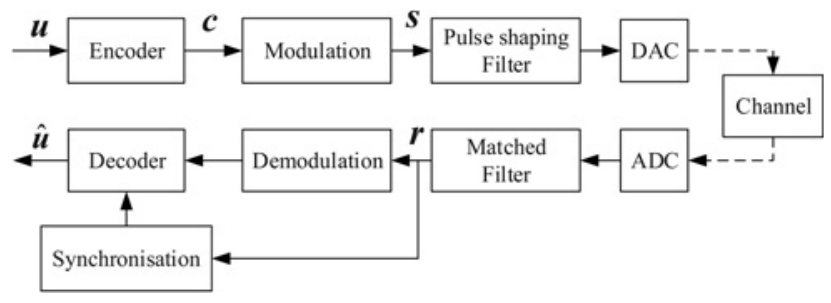

Fig. 1 Transceiver structure of general LR-WPANS

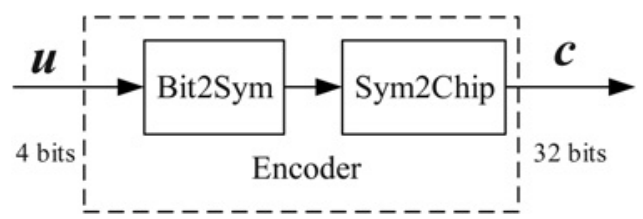

Fig. 2 Encoder of the 2.4 GHz PHY in IEEE 802.15.4 standard

Table 1 Symbol-to-chip mapping table in IEEE 802.15.4 standard

\begin{tabular}{lc}
\hline Data symbol & Chip values \\
$\left(u_{0}, u_{1}, u_{2}, u_{3}\right)$ & $\left(c_{0}, c_{1}, \ldots, c_{31}\right)$
\end{tabular}

00000

1000

0100

1100

00010

1010

0110

1110

11110

000

$\begin{array}{lllll}1 & 0 & 0 & 1\end{array}$

0101

11101

001

001

101

01111

1111

$\begin{array}{llllllllllllllllllllllllllllllll}1 & 1 & 0 & 1 & 1 & 0 & 0 & 1 & 1 & 1 & 0 & 0 & 0 & 0 & 1 & 1 & 0 & 1 & 0 & 1 & 0 & 0 & 1 & 0 & 0 & 0 & 1 & 0 & 1 & 1 & 1 & 0 \\ 1 & 1 & 1 & 0 & 1 & 1 & 0 & 1 & 1 & 0 & 0 & 1 & 1 & 1 & 0 & 0 & 0 & 0 & 1 & 1 & 0 & 1 & 0 & 1 & 0 & 0 & 1 & 0 & 0 & 0 & 1 & 0 \\ 0 & 0 & 1 & 0 & 1 & 1 & 1 & 0 & 1 & 1 & 0 & 1 & 1 & 0 & 0 & 1 & 1 & 1 & 0 & 0 & 0 & 0 & 1 & 1 & 0 & 1 & 0 & 1 & 0 & 0 & 1 & 0 \\ 0 & 0 & 1 & 0 & 0 & 0 & 1 & 0 & 1 & 1 & 1 & 0 & 1 & 1 & 0 & 1 & 1 & 0 & 0 & 1 & 1 & 1 & 0 & 0 & 0 & 0 & 1 & 1 & 0 & 1 & 0 & 1 \\ 0 & 1 & 0 & 1 & 0 & 0 & 1 & 0 & 0 & 0 & 1 & 0 & 1 & 1 & 1 & 0 & 1 & 1 & 0 & 1 & 1 & 0 & 0 & 1 & 1 & 1 & 0 & 0 & 0 & 0 & 1 & 1 \\ 0 & 0 & 1 & 1 & 0 & 1 & 0 & 1 & 0 & 0 & 1 & 0 & 0 & 0 & 1 & 0 & 1 & 1 & 1 & 0 & 1 & 1 & 0 & 1 & 1 & 0 & 0 & 1 & 1 & 1 & 0 & 0 \\ 1 & 1 & 0 & 0 & 0 & 0 & 1 & 1 & 0 & 1 & 0 & 1 & 0 & 0 & 1 & 0 & 0 & 0 & 1 & 0 & 1 & 1 & 1 & 0 & 1 & 1 & 0 & 1 & 1 & 0 & 0 & 1 \\ 1 & 0 & 0 & 1 & 1 & 1 & 0 & 0 & 0 & 0 & 1 & 1 & 0 & 1 & 0 & 1 & 0 & 0 & 1 & 0 & 0 & 0 & 1 & 0 & 1 & 1 & 1 & 0 & 1 & 1 & 0 & 1 \\ 1 & 0 & 0 & 0 & 1 & 1 & 0 & 0 & 1 & 0 & 0 & 1 & 0 & 1 & 1 & 0 & 0 & 0 & 0 & 0 & 0 & 1 & 1 & 1 & 0 & 1 & 1 & 1 & 1 & 0 & 1 & 1 \\ 1 & 0 & 1 & 1 & 1 & 0 & 0 & 0 & 1 & 1 & 0 & 0 & 1 & 0 & 0 & 1 & 0 & 1 & 1 & 0 & 0 & 0 & 0 & 0 & 0 & 1 & 1 & 1 & 0 & 1 & 1 & 1 \\ 0 & 1 & 1 & 1 & 1 & 0 & 1 & 1 & 1 & 0 & 0 & 0 & 1 & 1 & 0 & 0 & 1 & 0 & 0 & 1 & 0 & 1 & 1 & 0 & 0 & 0 & 0 & 0 & 0 & 1 & 1 & 1 \\ 0 & 1 & 1 & 1 & 0 & 1 & 1 & 1 & 1 & 0 & 1 & 1 & 1 & 0 & 0 & 0 & 1 & 1 & 0 & 0 & 1 & 0 & 0 & 1 & 0 & 1 & 1 & 0 & 0 & 0 & 0 & 0 \\ 0 & 0 & 0 & 0 & 0 & 1 & 1 & 1 & 0 & 1 & 1 & 1 & 1 & 0 & 1 & 1 & 1 & 0 & 0 & 0 & 1 & 1 & 0 & 0 & 1 & 0 & 0 & 1 & 0 & 1 & 1 & 0 \\ 0 & 1 & 1 & 0 & 0 & 0 & 0 & 0 & 0 & 1 & 1 & 1 & 0 & 1 & 1 & 1 & 1 & 0 & 1 & 1 & 1 & 0 & 0 & 0 & 1 & 1 & 0 & 0 & 1 & 0 & 0 & 1 \\ 1 & 0 & 0 & 1 & 0 & 1 & 1 & 0 & 0 & 0 & 0 & 0 & 0 & 1 & 1 & 1 & 0 & 1 & 1 & 1 & 1 & 0 & 1 & 1 & 1 & 0 & 0 & 0 & 1 & 1 & 0 & 0 \\ 1 & 1 & 0 & 0 & 1 & 0 & 0 & 1 & 0 & 1 & 1 & 0 & 0 & 0 & 0 & 0 & 0 & 1 & 1 & 1 & 0 & 1 & 1 & 1 & 1 & 0 & 1 & 1 & 1 & 0 & 0 & 0\end{array}$

finding new coding schemes with improved coding gain for LR-WPANs in this section. This paper is equivalent to finding a set of codewords with large minimum Hamming distance. It is known that the Griesmer bound [10], which is effective for codes with low code rate, provides an upper bound of linear block codes. With the Griesmer bound, the minimum Hamming distance $d_{\text {min }}$ of linear block codes is bounded by

$$
\sum_{i=0}^{k-1}\left\lceil\frac{d_{\min }}{2^{i}}\right\rceil \leq n
$$

where ' $\lceil\cdot\rceil$ ' denotes the smallest integer that is larger than or equal to the input. For $(32,4)$ linear block codes, Griesmer bound suggests that $d_{\min } \leq 16$. On the other hand, the bound table in [13] shows that linear block codes with $d_{\min }=16$ are possible. Our task is to find the codes that achieve the Griesmer bound.

We start the search with some well-studied linear block codes. One of the best-known codes, which are attractive for low-to-moderate block length, is $\mathrm{BCH}$ code. From the extensive list of $\mathrm{BCH}$ codes, we observe that $\mathrm{BCH}$ codes do not support the codeword length $n=32$. Instead, the $(31,6)$ primitive $\mathrm{BCH}$ code provides the largest minimum Hamming distance $d_{\min }=15$ with codeword length $n=31$ [12]. From [14], we know that by adding a parity-check bit, the $(n, k)$ binary linear block code with odd minimum Hamming distance $d_{\min }$ can be extended to a new $(n+$ $1, k)$ code. Meanwhile, the minimum Hamming distance of the extended code is also increased by 1 . By padding a parity-check bit to the $(31,6) \mathrm{BCH}$ code, the codeword length becomes 32 and the minimum Hamming distance of this code increases to 16 .

To design a bound-achieving $(32,4)$ block code that is compatible with the $2.4 \mathrm{GHz}$ PHY in IEEE 802.15.4 standard, we choose a subset of the $(32,6)$ extended $\mathrm{BCH}$ code corresponding to four independent information bits. A possible way to generate a $(32,4)$ code based on the $(32,6)$ extended BCH code is shown in Fig. 3. The input information bits, which are segmented into blocks of 4 bits, can be expressed as $\boldsymbol{u}=\left(u_{0}, u_{1}, u_{2}, u_{3}\right)$. In the 'Bit Pad' block, $\boldsymbol{u}$ is extended to $\boldsymbol{u}^{\prime}$ by padding two bits. These two bits can be padded in any position of $\boldsymbol{u}$. Valid combinations can be $\boldsymbol{u}^{\prime}=$ $\left(u_{0}, u_{1}, u_{2}, u_{3}, 0,1\right)$ or $\boldsymbol{u}^{\prime}=\left(u_{0}, u_{1}, u_{2}, 0, u_{3}, 1\right)$. To be complied with the IEEE 802.15.4 standard, we emphasise that the padded bits are not allowed to be all zeros. Since in this case,

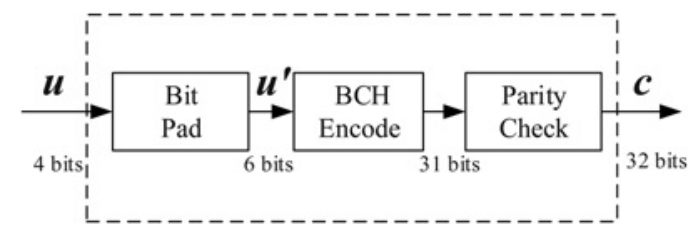

Fig. 3 (32,4) Code based on the (32,6) extended BCH code 


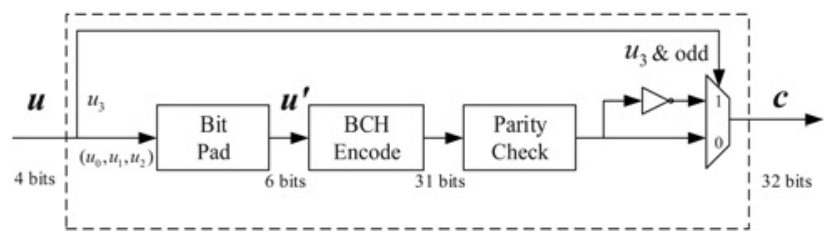

Fig. 4 Proposed $(32,4)$ code based on extended BCH code for LR-WPANS

all-zero or all-one codewords are possible. The mean transmit power may be different from other codewords. In addition, the pattern in all-zero or all-one codewords may interfere with other systems transmitting in the same frequency band. The 'BCH Encode' block encodes the 6 bit sequence $\boldsymbol{u}^{\prime}$ with the corresponding $(31,6)$ $\mathrm{BCH}$ code. At last, by adding a parity-check bit to the output of the $\mathrm{BCH}$ encoder, we obtain a 32 bit codeword $\boldsymbol{c}=\left(c_{0}, c_{1}, \ldots\right.$, $\left.c_{31}\right)$. The proposed coding scheme can be summarised in Fig. 3.

By studying the codewords of the $(32,6)$ extended $\mathrm{BCH}$ code, we observe that half of the 64 codewords are mutual complements of the other half. All chips between two complementary codewords are opposite. Each pair of such codewords has the largest Hamming distance 32 for the $(32,6)$ extended $\mathrm{BCH}$ code. The design of encoder and decoder can be further simplified by this property. Fig. 4 shows the updated $(32,4)$ block code. The four information bits $\boldsymbol{u}=\left(u_{0}, u_{1}, u_{2}, u_{3}\right)$ are separated into two parts: $\left(u_{0}, u_{1}, u_{2}\right)$ and $u_{3}$. In the 'Bit Pad' block, three bits are padded to $\left(u_{0}, u_{1}\right.$, $\left.u_{2}\right)$. One valid combination is $\boldsymbol{u}^{\prime}=\left(u_{0}, u_{1}, u_{2}, u_{3}=0,0,1\right)$. The corresponding $\mathrm{BCH}$ code and extended $\mathrm{BCH}$ code can be obtained. In this case, only eight codewords are chosen. The bit $u_{3}$ is utilised to generate the complementary codewords. When $u_{3}=1$, we first generate the corresponding codeword with $u_{3}=0$. Then, we flip all bits in the codeword to get the complementary codeword with a logic inverter. It is worth noting that these eight codewords are not generated from the $\mathrm{BCH}$ encoder and the parity-check bit extension. However, it is easy to show that these eight codewords are valid codewords satisfying the extended $\mathrm{BCH}$ code. Table 2 gives one possible symbol-to-chip mapping table of the proposed $(32,4)$ code. The proposed code has a minimum Hamming distance of 16 and performs better than the codewords used in the IEEE 802.15.4 standard.

The performance of the coding schemes for LR-WPANs can be further improved. With a given codeword length $n$, (1) indicates that the coding gain of block codes increases linearly with respect to the information bit length $k$ when the minimum Hamming distance $d_{\min }$ is the same. Since the $(32,6)$ extended BCH code achieves the same minimum Hamming distance $d_{\min }=16$ as the proposed $(32,4)$ code, the $(32,6)$ extended $\mathrm{BCH}$ code as shown in Fig. 5 can be directly applied in LR-WPANs to provide better coding gain. The information bits are segmented into blocks of 6 bits, which can be defined by $\boldsymbol{u}=\left(u_{0}, u_{1}, u_{2}, u_{3}, u_{4}, u_{5}\right)$.

Table 2 Symbol-to-chip mapping table of the proposed $(32,4)$ code based on extended $\mathrm{BCH}$ code

\begin{tabular}{|c|c|}
\hline $\begin{array}{l}\text { Data symbol } \\
\left(u_{0}, u_{1}, u_{2}, u_{3}\right)\end{array}$ & $\begin{array}{c}\text { Chip values } \\
\left(c_{0}, c_{1}, \ldots, c_{31}\right)\end{array}$ \\
\hline $\begin{array}{llll}0 & 0 & 0 & 0 \\
1 & 0 & 0 & 0 \\
0 & 1 & 0 & 0 \\
1 & 1 & 0 & 0 \\
0 & 0 & 1 & 0 \\
1 & 0 & 1 & 0 \\
0 & 1 & 1 & 0 \\
1 & 1 & 1 & 0 \\
0 & 0 & 0 & 1 \\
1 & 0 & 0 & 1 \\
0 & 1 & 0 & 1 \\
1 & 1 & 0 & 1 \\
0 & 0 & 1 & 1 \\
1 & 0 & 1 & 1 \\
0 & 1 & 1 & 1 \\
1 & 1 & 1 & 1\end{array}$ & 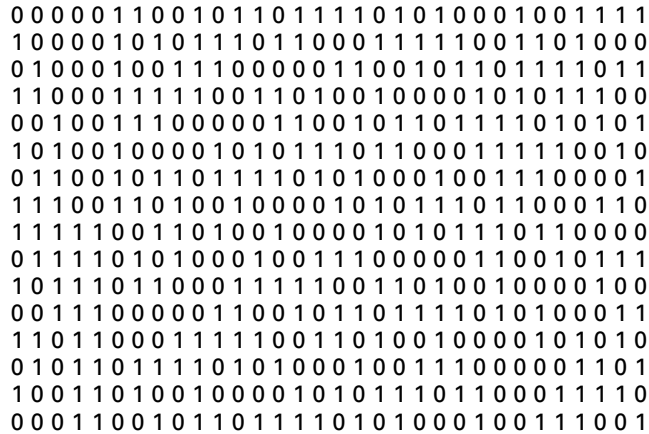 \\
\hline
\end{tabular}

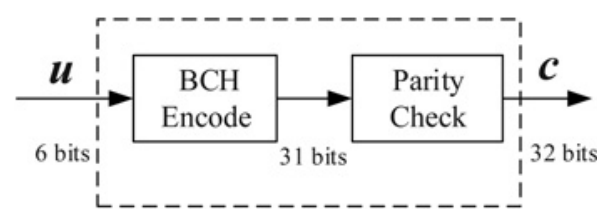

Fig. 5 (32,6) Extended BCH code for LR-WPANS

Similarly, we obtain a 32 bit codeword $c=\left(c_{0}, c_{1}, \ldots, c_{31}\right)$ by adding a parity-check bit after the $(31,6) \mathrm{BCH}$ encoder. To avoid all-zero or all-one codewords, a whitening block can be applied.

Next, we will discuss the decoder of the proposed coding schemes. In short-range communications such as IEEE 802.15.4, AWGN channel is considered to be the general case [15]. At the receiver side, with perfect synchronisation, the received baseband signal can be represented as

$$
r_{i}=s_{i}+v_{i}
$$

where $r_{i}$ is the received baseband signal at the ideal sampling time, $s_{i}$ is the $i$ th chip modulated with OQPSK in a 32-chip sequence and $v_{i}$ is the equivalent AWGN with variance $\sigma^{2}$. Since the proposed $(32,4)$ code is based on the $(32,6)$ extended $\mathrm{BCH}$ code, existing decoding algorithms of $\mathrm{BCH}$ codes are available. However, the performance of these hard-decision decoding algorithms is not satisfactory. With a coherent demodulator for the OQPSK system [8], the information is only contained in one branch and the noise in the other branch can be completely ignored. The $i$ th chip in a symbol for coherent demodulator can be described as

$$
r_{i}^{\prime}=\left\{\begin{array}{lc}
\operatorname{Re}\left\{r_{\mathrm{i}}\right\}=s_{i}^{\prime}+\operatorname{Re}\left\{v_{i}\right\}, & (i \bmod 2)=0, \\
\operatorname{Im}\left\{r_{\mathrm{i}}\right\}=s_{i}^{\prime}+\operatorname{Im}\left\{v_{i}\right\}, & \text { otherwise },
\end{array}\right.
$$

where $\operatorname{Re}\{x\}$ is the real part of $x, \operatorname{Im}\{x\}$ is the imaginary part of $x$ and $s_{i}^{\prime} \in\{-1,+1\}$ is the $i$ th chip modulated with binary phase shift keying. For a certain codeword $\boldsymbol{c}$, the corresponding mapped chip sequence would be $\boldsymbol{s}^{\prime}=\left(s_{0}^{\prime}, s_{1}^{\prime}, s_{2}^{\prime}, \ldots, s_{31}^{\prime}\right)$. Since $\operatorname{Re}\left\{v_{i}\right\}$ and $\operatorname{Im}\left\{v_{i}\right\}$ are Gaussian noises with variance $\sigma^{2} / 2$, the conditional probability density function of received bit $r_{i}^{\prime}$ given a prior information of transmitted bit $s_{i}^{\prime}$ can be estimated by

$$
f\left(r_{i}^{\prime} \mid s_{i}^{\prime}\right)=\frac{1}{\sqrt{\pi \sigma^{2}}} \mathrm{e}^{-\left(\left|r_{i}^{\prime}-s_{i}^{\prime}\right|^{2} / \sigma^{2}\right)}
$$

The conditional probability density function of the received 32-chip sequence given a prior information of the transmitted chip sequence $\boldsymbol{s}^{\prime}$ can be estimated by

$$
\begin{aligned}
f\left(\boldsymbol{r}^{\prime} \mid \boldsymbol{s}^{\prime}\right) & =\prod_{i=0}^{31} \mathrm{e}^{-\left|r_{i}^{\prime}-s_{i}^{\prime}\right|^{2}} \\
& =\left(\prod_{i=0}^{31} \mathrm{e}^{-\left(\left|r_{i}^{\prime}\right|^{2}+1\right)}\right) \times \mathrm{e}^{\sum_{i=0}^{31} 2 r_{i}^{\prime} \cdot s_{i}^{\prime}}
\end{aligned}
$$

where $\boldsymbol{r}^{\prime}=\left(r_{0}^{\prime}, r_{1}^{\prime}, r_{2}^{\prime}, \ldots, r_{31}^{\prime}\right)$ is the received soft chip sequence. In (6), the term $\prod_{i=0}^{31} \mathrm{e}^{-\left(\left|r_{i}^{\prime}\right|^{2}+1\right)}$ is a constant for all possible codewords $c$. The term $\sum_{i=0}^{31} 2 r_{i}^{\prime} \cdot s_{i}^{\prime}$ is a real number. Since $\mathrm{e}^{x}$ is a monotonically increasing function when $x$ is real, the maximum-likelihood (ML) sequence decoder of the above block code can be written as

$$
\begin{aligned}
\hat{\boldsymbol{c}} & =\underset{\boldsymbol{c}}{\arg \max } f\left(\boldsymbol{r}^{\prime} \mid \boldsymbol{s}^{\prime}\right) \\
& =\underset{\boldsymbol{c}}{\arg \max } \sum_{i=0}^{31} r_{i}^{\prime} \cdot s_{i}^{\prime} .
\end{aligned}
$$


Equation (7) suggests that the optimal cross-correlation between the received soft chips and the modulated codeword gives the ML sequence decoder of the codeword.

By adopting the proposed $(32,4)$ code defined in Fig. 4, we can further simplify the ML sequence decoder by computing only half of the correlation values since half of the codewords are just the opposite values of the other codewords.

At the end of this section, we would like to discuss the computational complexity of the proposed block coding schemes. Comparing the proposed $(32,4)$ code with the code used in the standard (as shown in Table 1), a simple look-up-table (LUT) can be applied as the encoder. The codewords used in the standard have cyclic shift structure that can be applied to further simplify the encoder design. Nevertheless, the encoder complexities of both codes are low. For the proposed $(32,6)$ code, the LUT-based encoder is slightly more complex than the code used in the standard. On the other hand, if we generate the codewords from the $\mathrm{BCH}$ generator polynomial, the encoder of the $(32,6)$ code can also be simple.

In the decoder, the above proposed ML sequence decoder achieves optimal symbol error rate for the proposed block codes as well as the code used in the standard. As we discussed earlier, for the proposed $(32,4)$ code, we only need to compute eight cross-correlation values between received soft chips and the candidate codewords. Given the properties of the code used in the standard, we conclude that the optimal decoder also needs to compute eight cross-correlation values. Thus, the decoding complexities of these two codes are about the same. For the proposed $(32,6)$ extended $\mathrm{BCH}$ code, 32 cross-correlation values need to be computed. The decoding complexity is slightly higher than the $(32,4)$ code. In summary, the proposed block code provides additional coding gain to the LR-WPANs systems with no or little additional computational complexity.

\section{Coding schemes based on convolutional codes}

In Section 3, we propose the improved coding schemes based on linear block codes. The proposed coding schemes are similar to that used in current standard and require minimum changes to the standard. The proposed coding schemes achieve the upper bound of $d_{\min }$ as linear block codes. In practice, the coding gain can be further improved if we do not restrict the type of codes. One of the alternative codes is convolutional code. In this section, we discuss the possibility of applying convolutional codes to LR-WPANs. Optimum generator polynomials of convolutional codes are utilised for LR-WPANs.

In general, we assume that the encoder has a code rate $R_{\mathrm{c}}=1 / n$ with constraint length $K$ in LR-WPANs. The asymptotic coding gain for convolutional codes with soft decision in AWGN channel can be defined as

$$
\gamma \simeq 10 \log _{10}\left(R_{\mathrm{c}} d_{\mathrm{f}}\right)
$$

where $d_{\mathrm{f}}$ is the free distance of convolutional codes. For a given code rate $R_{\mathrm{c}}$, the asymptotic coding gain is restricted by the free distance of convolutional codes. The free distance of convolutional codes is directly related to the generator polynomials. The Heller bound or the upper bound of the free distance of convolutional codes with code rate $R_{\mathrm{c}}=1 / n$ is given by [11]

$$
d_{\mathrm{f}} \leq d_{\mathrm{H}} \triangleq \min _{l \geq 1}\left\lfloor\frac{2^{l-1}}{2^{l}-1}(K+l-1) n\right\rfloor,
$$

where ' $\lfloor\cdot\rfloor$ ' denotes the largest integer that is less than or equal to the input. Assume that $d_{\mathrm{H}}$ is minimised at $l=h$, that is, $d_{\mathrm{H}}=\min _{h \geq 1}\left\lfloor\left(2^{h-1} / 2^{h}-1\right)(K+h-1) n\right\rfloor$. Then, a tighter bound can be obtained when $d_{\mathrm{H}}$ is odd and $d_{\mathrm{H}}>\left(2^{h-1} / 2^{h}-1\right)\{(K+h-1)$ $\left.n-1+2^{1-h}\right\}$. We have

$$
d_{\mathrm{f}} \leq \min _{l \geq 1}\left\lfloor\frac{2^{l-1}}{2^{l}-1}(K+l-1) n\right\rfloor-1 .
$$

Exhaust search of generator polynomials, which can be terminated when the free distance of encoder achieves the improved Heller bound, has been executed to find maximum free distance convolutional codes [12]. The maximum free distance convolutional codes are often deemed as optimum convolutional codes in AWGN channel. Frenger et al. [16] presented a family of rate-compatible LR convolutional codes with maximum free distance. Maximum free distance codes with rates ranging from 1/ 4 to $1 / 512$ and constraint lengths between 3 and 11 are tabulated in [16]. With these codes, a convolutional code of any code rate $R_{\mathrm{c}}=1 / n$ can be obtained for different constraint lengths, resulting in flexible and powerful coding schemes. In this paper, we utilise the existing LR convolutional codes for further discussion.

In LR-WPANs, the packet length is usually small. The application of convolutional codes may result in rate loss when transmitting additional known terminating bits to flush out the memory. TBC codes can help to eliminate the rate loss by initialising the memory state with actual tail bits in the transmitted sequence. It is widely used in numerous communication systems such as the worldwide interoperability for microwave access and the long-term evolution. In IEEE 802.15.4 standard, the maximum length of payload is as small as 20 bytes. The TBC codes with optimum generator polynomials are suitable coding schemes for short-packet applications.

Let $C$ be an $(n, 1)$ TBC code, where $n$ is the number of output bits per input information bit. The encoder of TBC code is initialised with the tail $K-1$ information bits in the transmitted sequence, where $K$ is the constraint length of the code. The output bits per information bit are generated corresponding to memory state and generator polynomials, which can be defined by $c=\left(c_{0}, c_{1}, \ldots\right.$, $\left.c_{n-1}\right)$.

At the receiver side, many studies have been made to reduce the decoding complexity of the ML decoders for tail-biting trellises [17-19]. Sub-optimal decoders such as wrap-around Viterbi algorithm [20] and other alternatives [21] are also developed. Since decoding algorithms of the TBC code are not the focus of this paper, we select a low-complexity sub-optimal method in [21] to decode the TBC code for LR-WPANs. Denote $\boldsymbol{r}^{\prime}=\left(r_{0}^{\prime}, r_{1}^{\prime}, \ldots, r_{n L-1}^{\prime}\right)$ as the received soft chip sequence, where $L$ is the length of the information sequence. Copying the ending $n T$ values of $\boldsymbol{r}^{\prime}$ to the front of the received sequence, and copying the leading $n H$ values of $\boldsymbol{r}^{\prime}$ to the end of the received sequence, the extended received sequence for TBC code decoder is given by

$\boldsymbol{r}_{\mathrm{e}}=\left(r_{n L-1-(n T-1)}^{\prime}, \ldots, r_{n L-1}^{\prime}, r_{0}^{\prime}, r_{1}^{\prime}, \ldots, r_{n L-1}^{\prime}, r_{0}^{\prime}, \ldots, r_{n H-1}^{\prime}\right)$,

where $T$ is the length of copied information bits in the tail and $H$ is the length of copied information bits in the head. A super trellis can be constructed on $\boldsymbol{r}_{\mathrm{e}}$. It is safe to assume that the starting and terminating states are known as all-zero states for this trellis. Viterbi algorithm can be applied to the super trellis $\boldsymbol{r}_{\mathrm{e}}$. The corresponding decoder output of the middle $L$ bits is the decoder output. The performance loss of this sub-optimal decoder is negligible when $T \geq 5 K$ and $H \geq 5 K$ [21].

In IEEE 802.15.4 standard, the chip rate is eight times to that of information bits, which can be viewed as an encoder with code rate $R_{\mathrm{c}}=1 / 8$. For a given code rate $R_{\mathrm{c}}=1 / 8$, the maximum free distance of improved Heller bound is shown in Fig. 6. The maximum free distance increases almost linearly with constraint length $K$. However, the number of states in trellis, which indicates the complexity of decoding algorithm, increases exponentially with the constraint length of the code. The constraint length $K=7$ is chosen as a compromise between performance and complexity 


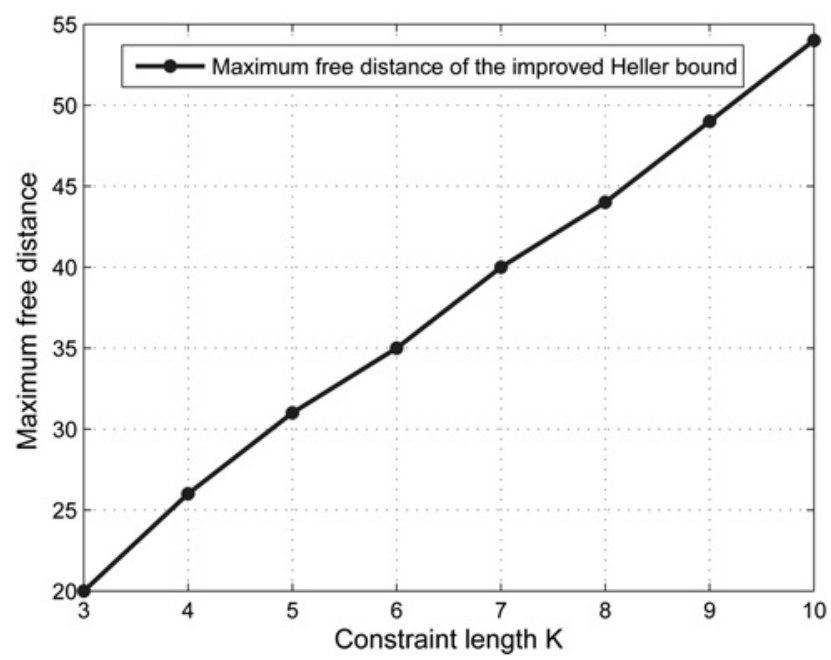

Fig. 6 Maximum free distance of the improved Heller bound with code rate $R_{c}=1 / 8$

$[22,23]$. The corresponding generator polynomials of TBC code in octal format are 117, 127, 135, 135, 145, 155, 171, 173 [16].

Next, we evaluate the encoding and decoding complexities of the proposed TBC code. The encoder of the TBC involves the operation of shift registers with length 6 . The encoder complexity is low. In the decoder part, the sub-optimal reduced complexity circular Viterbi decoding algorithm [21] gives satisfactory performance with comparable complexity as the regular Viterbi algorithm. Such decoder has higher computational complexity than the ML sequence decoder for the block code. However, the computational complexity of the Viterbi-like algorithm is still acceptable for LR-WPAN systems with reasonable increase of the logic size and memory size. We emphasise that the coding gain of the proposed TBC code is significant comparing with the block code in the standard.

\section{Simulation results}

In this section, we explore the PER performance of the proposed coding schemes. The transceiver structure is shown in Fig. 1. The encoder block takes random binary input and generates coded bits. The coded bits are mapped to constellations with OQPSK modulation. A half-sine pulse shaping filter is applied in the transmitter. AWGN channel and Rayleigh flat-fading channel are utilised in the following simulations. At the receiver side, matched filter is utilised to maximise the SNR of the received signals. Perfect synchronisation is assumed. For the block code, the ML decoder in (7) is applied. For the TBC code, a low-complexity sub-optimal Viterbi algorithm [21] is utilised for decoding. The detailed description of the decoding algorithm is listed in Section 4. The system performance is evaluated at PER of $1 \%$ with effective payload length of 20 bytes. The number of simulated packets is $10^{7}$ in every SNR condition. The simulation setup is summarised in Table 3.

In the first simulation, we compare the PER performance of the $(32,4)$ code used in current standard, the $(32,4)$ code based on

Table 3 Simulation parameters

\begin{tabular}{lc}
\hline Parameters & Values \\
\hline modulation & OQPSK \\
pulse shaping filter & half-sine \\
chip rate & $2 \mathrm{Mb} / \mathrm{s}$ \\
payload length & $20 \mathrm{bytes}$ \\
number of packets & $10^{7}$ \\
\hline
\end{tabular}

extended $\mathrm{BCH}$ code, the $(32,6)$ extended $\mathrm{BCH}$ code and the TBC code with optimum generator polynomials in AWGN channel.

Fig. 7 shows the PER performance of different coding schemes in AWGN channel. The PER performance with respect to SNR per bit is shown in Fig. 7a. For LR-WPANs, the receive sensitivity is usually measured by SNR per chip. We redraw Fig. $7 a$ in Fig. $7 b$ to show the PER performance with respect to SNR per chip. The dashed lines show PER performance of the $(32,4)$ code used in current standard; the solid lines show PER performance of the $(32,4)$ code based on extended $\mathrm{BCH}$ code; the dash-dotted lines show PER performance of the $(32,6)$ extended $\mathrm{BCH}$ code; and the dotted lines show PER performance of the TBC code with optimum generator polynomials. From Fig. $7 a$, we observe that at the PER of $1 \%$, the $(32,4)$ code based on extended $\mathrm{BCH}$ code achieves about $0.5 \mathrm{~dB}$ coding gain compared with the $(32,4)$ code used in current standard. The $(32,6)$ extended $\mathrm{BCH}$ code achieves about $1.8 \mathrm{~dB}$ coding gain compared with the $(32,4)$ code used in current standard. While the TBC code with optimum generator polynomials achieves about $4.3 \mathrm{~dB}$ coding gain compared with the $(32,4)$ code used in current standard.

In Fig. $7 b$, the $x$-axis changes to SNR per chip, which denotes the receive sensitivity in practice. Since the $(32,4)$ code based on extended $\mathrm{BCH}$ code and the $(32,4)$ code used in current standard have the same rate, the performance gain is $0.5 \mathrm{~dB}$ in terms of the sensitivity as well. We would like to emphasise that the encoding
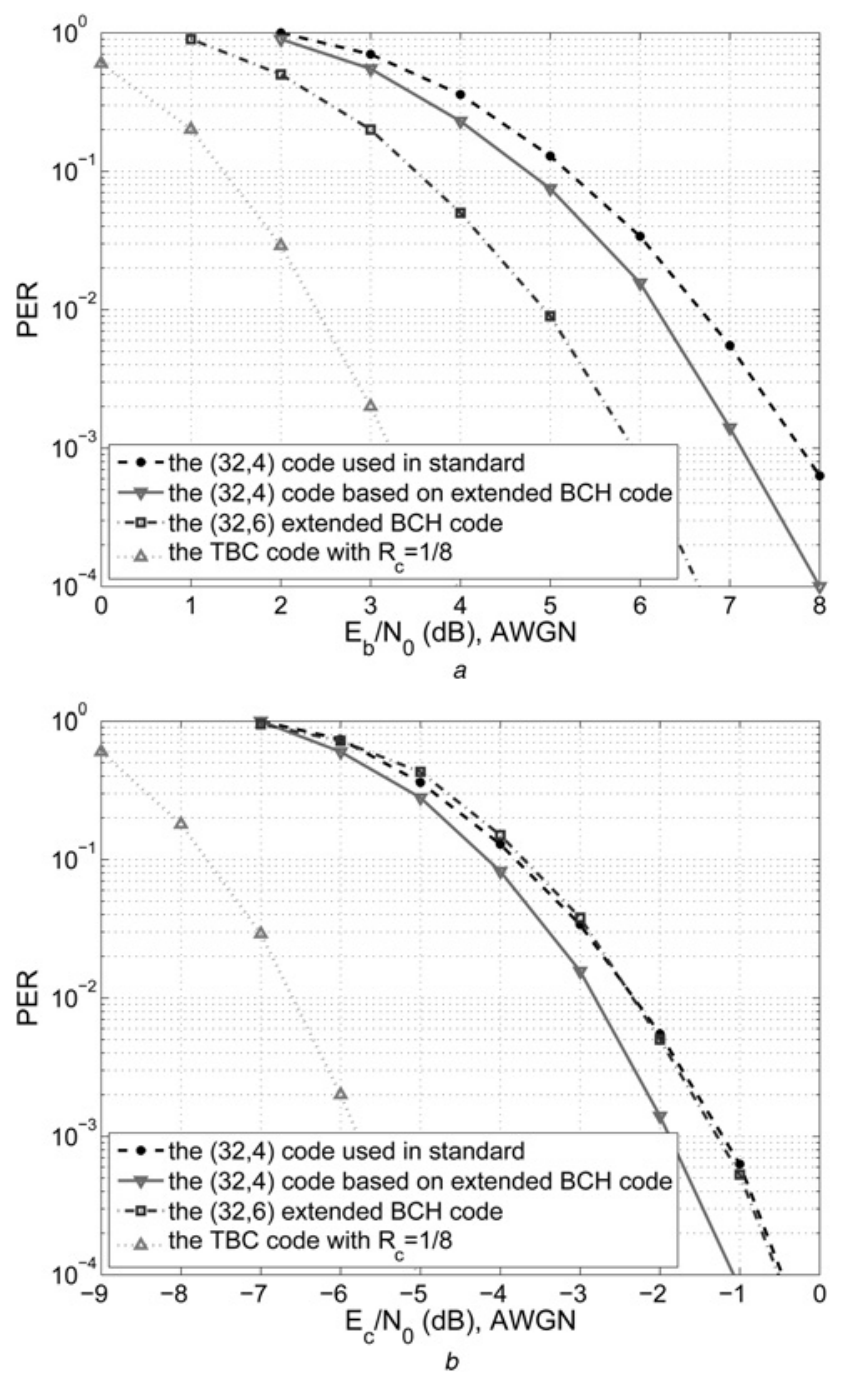

Fig. 7 PER performance of the proposed codes in AWGN channel $a$ PER performance of the proposed codes with respect to SNR per bit in AWGN channel

$b$ PER performance of the proposed codes with respect to SNR per chip in AWGN channel 


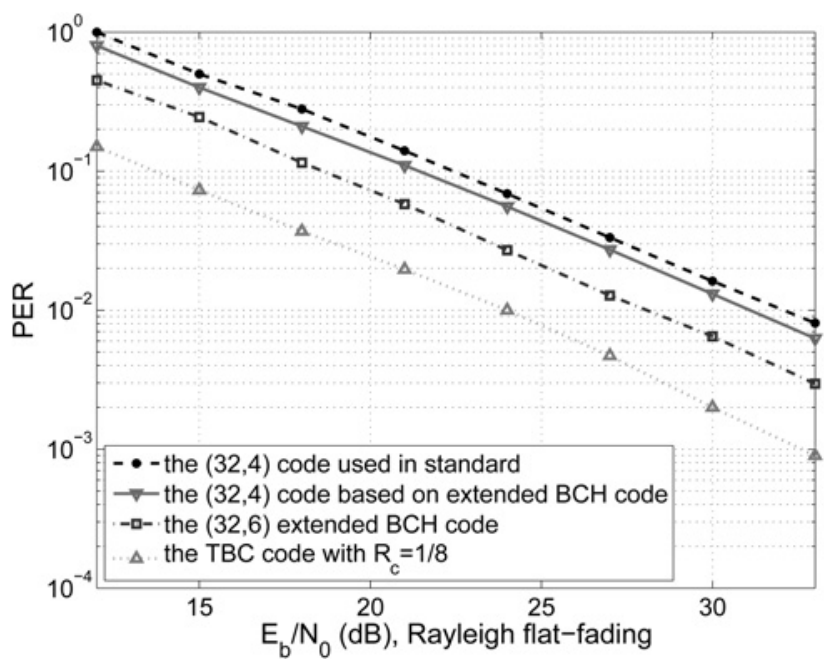

Fig. 8 PER performance of the proposed codes in Rayleigh flat-fading channel

and decoding complexities of the two codes are about the same. The performance of the $(32,6)$ extended $\mathrm{BCH}$ code is about the same as that of the $(32,4)$ code used in current standard. However, with the $(32,6)$ extended $\mathrm{BCH}$ code, the data rate improves by $50 \%$. The performance improvement of TBC code is significant. The above three coding schemes have their specific advantages. The $(32,4)$ code based on extended $\mathrm{BCH}$ code improves performance without extra complexity. The $(32,6)$ extended $\mathrm{BCH}$ code improves the data rate without receive sensitivity loss. The TBC code with optimum generator polynomials significantly improves the performance at the expense of extra decoding complexity.

In the second simulation, we repeat the simulation in Rayleigh flat-fading channel. The PER performance with respect to SNR per bit is shown in Fig. 8. The definition of each line in Fig. 8 follows the definition in the first simulation. We observe that the $(32,4)$ code based on extended $\mathrm{BCH}$ code, the $(32,6)$ extended $\mathrm{BCH}$ code and the TBC code with optimum generator polynomials achieves about 1.0, 4.0 and $8.0 \mathrm{~dB}$ coding gain compared with the $(32,4)$ code used in current standard, respectively. The performance improvement in Rayleigh flat-fading channel is even more significant than that in AWGN channel.

\section{Discussion}

The purpose of this paper is to provide solutions in improving the coding gain of LR-WPANs. Our proposed coding schemes can be applied to other LR-WPANs standards as well. For example, IEEE 802.15.4c standard has similar coding scheme to IEEE 802.15.4 standard. Every four information bits $\left(u_{0}, u_{1}, u_{2}, u_{3}\right)$ are mapped to a 16-chip sequence [24]. The minimum Hamming distance of this $(16,4)$ code is $d_{\min }=6$. This coding scheme does not fully utilise the benefit of the code redundancy either. By adopting similar coding scheme as shown in Fig. 4, we design a $(16,4)$ code based on extended $\mathrm{BCH}$ code, which achieves the upper bound of linear block $(16,4)$ code with $d_{\min }=8$. The $(16,5)$ extended $\mathrm{BCH}$ code is found to improve the data rate without performance loss. In addition, the optimum generator polynomials of the TBC code in octal format with code rate $R_{\mathrm{c}}=1 / 4$ and constraint length $K=7$ are $117,127,155,171$ [16].

Fig. 9 shows the performance with respect to SNR per bit of the $(16,4)$ code used in IEEE $802.15 .4 \mathrm{c}$ standard, the $(16,4)$ code based on extended BCH code, the $(16,5)$ extended BCH code and the TBC code with optimum generator polynomials in AWGN channel. The simulation setup is similar to that in Section 5. The definition of each line follows the definition in Fig. 7. Compared with the $(16,4)$ code used in IEEE 802.15.4c standard, the performance gains of the $(16,4)$ code based on extended $\mathrm{BCH}$

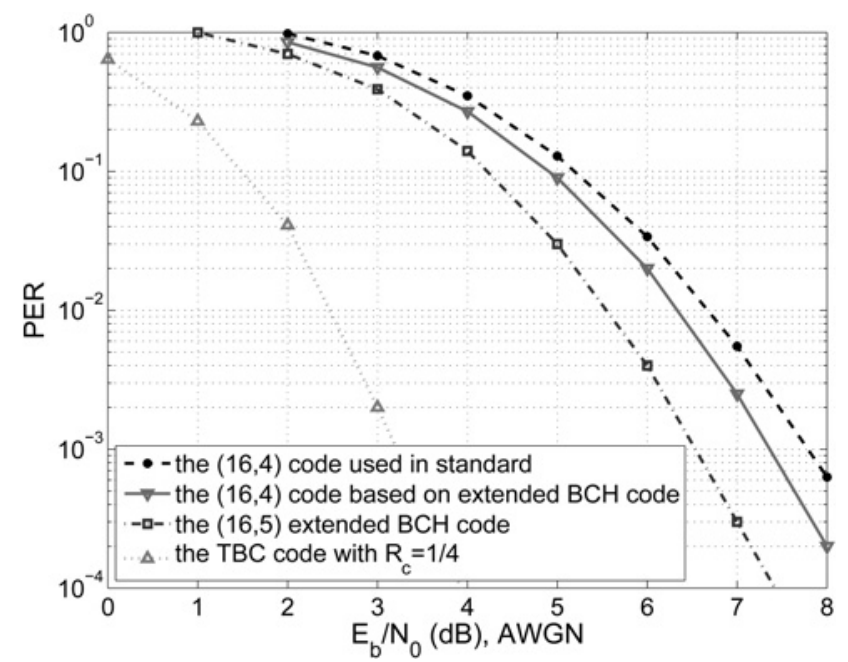

Fig. 9 PER performance of the proposed codes for IEEE 802.15.4C standard in AWGN channel

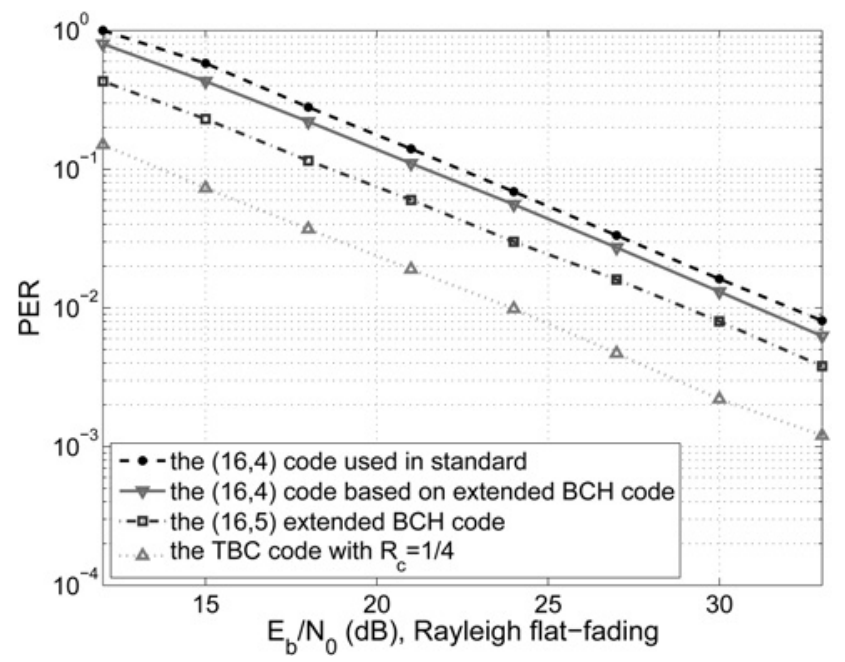

Fig. 10 PER performance of the proposed codes for IEEE 802.15.4C standard in Rayleigh flat-fading channel

code, the $(16,5)$ extended $\mathrm{BCH}$ code and the TBC code with optimal generator polynomials are $0.4,0.9$ and $4.1 \mathrm{~dB}$, respectively.

Similarly, Fig. 10 shows the performance with respect to SNR per bit of the $(16,4)$ code used in IEEE 802.15.4c standard, the $(16,4)$ code based on extended $\mathrm{BCH}$ code, the $(16,5)$ extended $\mathrm{BCH}$ code and the TBC code with optimum generator polynomials in Rayleigh flat-fading channel. The definition of each line follows the definition in Fig. 7. Compared with the $(16,4)$ code used in IEEE $802.15 .4 \mathrm{c}$ standard, the performance gains of the $(16,4)$ code based on extended $\mathrm{BCH}$ code, the $(16,5)$ extended $\mathrm{BCH}$ code and the TBC code with optimal generator polynomials are 1.0, 3.0 and $8.0 \mathrm{~dB}$, respectively.

\section{Conclusion}

In LR-WPANs, existing coding techniques do not fully utilise the benefit of the code redundancy. In this paper, we propose new coding schemes that achieve the largest minimum Hamming distance $d_{\text {min }}$ or free distance $d_{\mathrm{f}}$ at the required code rate. In IEEE 802.154 system, the proposed $(32,4)$ code achieves about $0.5 \mathrm{~dB}$ improvement in terms of PER performance in AWGN channel and $1.0 \mathrm{~dB}$ in Rayleigh flat-fading channel. In addition, the design 
complexities of encoder and decoder remain about the same. The coding gain of the $(32,6)$ extended $\mathrm{BCH}$ code is $1.8 \mathrm{~dB}$ in AWGN channel and $4.0 \mathrm{~dB}$ in Rayleigh flat-fading channel. With the $(32,6)$ extended $\mathrm{BCH}$ code, the data rate can be increased by $50 \%$ with no performance loss. The TBC code with optimum generator polynomials significantly improves the system performance by 4.3 $\mathrm{dB}$ in AWGN channel and $8.0 \mathrm{~dB}$ in Rayleigh flat-fading channel. The proposed coding schemes can be extended to other LR-WPANs standards.

\section{Acknowledgments}

This work was supported in part by the National Natural Science Foundation of China (no. 61231009), the National Key Science and Technology 863 Project (no. SS2015AA011303), the Science and Technology Commission Foundation of Shanghai (no. 14511100200), and the State Key Laboratory of Rail Traffic Control and Safety (no. RCS2014K004), Beijing Jiaotong University.

\section{References}

1 Kranenburg, R., Anzelmo, E., Bassi, A., et al.: 'The Internet of things'. Proc. First Berlin Symp. on Internet and Society, October 2011, pp. 25-27

2 IEEE Std. 802.15.4-2006: 'IEEE standard for information technology - part 15.4: wireless medium access control (MAC) and physical layer (PHY) specifications for low-rate wireless personal area networks (WPANs)', 2006

3 Wang, C., Huang, J.: 'A low-power $2.45 \mathrm{GHz}$ ZigBee transceiver for wearable personal medical devices in WPAN'. Proc. IEEE Int. Conf. on Consumer Electronics (ICCE'2007), 2007, pp. 1-2

4 Wang, C., Sung, G., Huang, J., et al.: 'A low-power $2.45 \mathrm{GHz}$ WPAN modulator/ demodulator', Microelectron. J., 2010, 41, (2), pp. 150-154

5 Yu, Y., Meng, X., Xiao, S., et al.: 'A new low-cost demodulator for $2.4 \mathrm{GHz}$ ZigBee receivers', J. Electron. (China), 2009, 26, (2), pp. 252-257

6 Yin, S., Cui, J., Luo, A., et al.: 'A high efficient baseband transceiver for IEEE 802.15.4 LR-WPAN systems'. Proc. IEEE Ninth Int. Conf. on ASIC, 2011, pp. $224-227$
7 Teng, C., Fonseka, J., Dowling, E.: 'Non-coherent detectors for quadrature-multiplexed continuous phase modulation signals', IET Commun., 2009, 3, (4), pp. 610-619

8 Dai, S., Qian, H., Kang, K., et al.: 'A robust demodulator for OQPSK-DSSS system', Circuits Syst. Signal Process., 2015, 34, (1), pp. 231-247

9 Lanzisera, S., Pister, K.: 'Theoretical and practical limits to sensitivity in IEEE 802.15.4 receivers'. Proc. IEEE Int. Conf. on Electronics, Circuits and Systems (ICECS'2007), 2007, pp. 1344-1347

10 Griesmer, J.: 'A bound for error-correcting codes', IBM J. Res. Dev., 1960, 4, (5), pp. 532-542

11 Daut, D., Modestino, J., Wismer, L.: 'New short constraint length convolutional code constructions for selected rational rates (Corresp.)', IEEE Trans. Inf. Theory, 1982, 28, (5), pp. 794-800

12 Proakis, J.: 'Digital communications' (McGraw-Hill, New York, 2007, 5th edn.)

13 'Bounds on the minimum distance of linear codes and quantum codes'. Available at http://www.codetables.de, 2007

14 Simonis, J.: 'Adding a parity-check bit', IEEE Trans. Inf. Theory, 2000, 46, (4), pp. $1544-1545$

15 Lee, S., Kwon, H., Jung, Y., et al.: 'Efficient non-coherent demodulation scheme for IEEE 802.15.4 LR-WPAN systems', Electron. Lett., 2007, 43, (16), pp. $879-880$

16 Frenger, P., Orten, P., Ottosson, T.: 'Code-spread CDMA using maximum free distance low-rate convolutional codes', IEEE Trans. Commun., 2000, 48, (1), pp. 135-144

17 Qian, H., Wang, X., Kang, K.: 'A depth-first ML decoding algorithm for tail-biting trellises', IEEE Trans. Veh. Technol., 2014, 64, (8), pp. 3339-3346

18 Pai, H., Han, Y., Wu, T.: 'Low-complexity ML decoding for convolutional tail-biting codes', IEEE Commun. Lett., 2008, 12, (12), pp. 883-885

19 Wang, X., Qian, H., Xiang, W.: 'An efficient ML decoder for tail-biting codes based on circular trap detection', IEEE Trans. Commun., 2013, 61, (4), pp. $1212-1221$

20 Shao, R., Lin, S., Fossorier, M.: 'Two decoding algorithms for tailbiting codes', IEEE Trans. Commun., 2003, 51, (10), pp. 1658-1665

21 Sung, W.: 'Minimum decoding trellis lengths for tail-biting convolutional codes', Electron. Lett., 2000, 36, (7), pp. 643-645

22 IEEE Std. 802.16-2009: 'IEEE standard for local and metropolitan area networks part 16: air interface for broadband wireless access systems', 2009

23 3GPP TS 36.212: '3rd generation partnership project; technical specification group radio access network; evolved universal terrestrial radio access (E-UTRA); multiplexing and channel coding (Release 8)', 2007

24 IEEE Std. 802.15.4c-2009: 'IEEE standard for information technology - part 15.4 wireless medium access control (MAC) and physical layer (PHY) specifications for low-rate wireless personal area networks (WPANs)', 2009 